\title{
INTERACTION BETWEEN In IMPURITY ATOMS AND IMPURITY ATOMS OF Mn AND Co DISSOLVED IN SILVER
}

\author{
M. Sternik and K. Królas \\ Institute of Physics, Jagiellonian University, Reymonta 4, 30-059 Kraków, Poland
}

(Received July 17, 1992)

\begin{abstract}
The interaction between impurities of two solute elements in metal hosts leads to a non-random distribution of impurity atoms among the lattice sites. Experimental studies of this distribution provide quantitative information on the energy involved in the formation of an individual impurity-impurity pair. The perturbed angular correlation measurements performed with ${ }^{111}$ In probe atoms and Mössbauer spectra of ${ }^{57} \mathrm{Co}$ are used to derive the interaction energy of In atoms with $\mathrm{Mn}$ and Co atoms introduced into silver matrix. It is demonstrated that In atoms attract $\mathrm{Mn}$ and Co atoms and in the case of In-Mn pair the interaction energy value was determined. The obtained results are discussed in the frame of the semiempirical model and the recent first principles theoretical calculations.
\end{abstract}

PACS numbers: $61.70 .-\mathrm{r}, 76.80 .+\mathrm{y}$

\section{Introduction}

The hyperfine interaction techniques such as perturbed angular correlation (PAC) and Mössbauer spectroscopy offer a possibility of studying local environments of radioactive probe atoms introduced into solids. In the case of dilute alloys, measurements of the hyperfine interaction spectra yield a direct insight into the structure of the first shell of the decaying atom. If a chemical preference exists for the probe to become surrounded with the impurity rather than host atoms the nearest neighbour probe-impurity pairs are formed more frequently than expected for a random constituent distribution. From the observed fraction of probe atoms bound to impurity atoms one can draw conclusions about the energy involved in the impurity-pair interaction.

The aim of the experiment described here is to continue measurements of the binding energy for impurity-impurity pairs in silver. The experimental results which were reviewed at the last Hyperfine Interactions Conference [1] have 
stimulated a new approach in the theoretical calculations. Hoshino et al. [2] presented their $a b$ initio calculations based on the local-density theory using the Korringa-Kohn-Rostoker (KKR) Green's function method for spherical potentials. The authors were able to reproduce successfully the available experimental interaction energies for several individual impurity-impurity pairs in silver and palladium hosts. Another fruitful test for the theory comes from the comparison of the theoretical and experimental results for a long series of elements being the impurity atoms which interact with the same probe in the same host.

In our systematic study we kept the ${ }^{111}$ In PAC probe and the host of silver constant and measured the interaction between ${ }^{111} \mathrm{In}$ and impurity atoms of the fourth period elements. It was demonstrated that the interaction changes its character from strongly repulsive for In-Ga and In-Zn impurity pairs to weakly attractive for $\mathrm{In}-\mathrm{Cu}$ and strongly attractive for In-Ni impurity pairs [3, 4]. In the present work we extended these measurements to the interaction between In-Co and In-Mn impurity pairs in silver. From two constituents of the pair, in the former case indium was the alloying element while cobalt as the ${ }^{57} \mathrm{Co}$ Mössbauer effect radioactive probe was added to the system. In the latter case, ${ }^{111}$ In was the radioactive $\mathrm{PAC}$ probe and manganese was the alloying element.

\section{2. ${ }^{111}$ In in AgMn dilute alloys}

The alloy $\mathrm{Ag}_{0.99} \mathrm{Mn}_{0.01}$ was prepared by arc-melting pure components: $(6 \mathrm{~N}) \mathrm{Ag}$ and $(4 \mathrm{~N}) \mathrm{Mn}$. More dilute alloys containing 0.5 and 0.2 at.\% of Mn were produced by co-melting the master alloy with appropriate amount of silver. The alloys were cold rolled onto thin foils and irradiated with $27 \mathrm{MeV} \alpha$-particles at the Cracow cyclotron. The radioactive ${ }^{111}$ In was produced in targets as the result of ${ }^{109} \mathrm{Ag}(\alpha, 2 \mathrm{n}){ }^{111} \mathrm{In}$ nuclear reaction. The samples were sealed in quartz tubes under an argon atmosphere and mounted in a small oven in a $\gamma-\gamma$ angular correlation apparatus. The PAC measurements were performed in the temperature region from $500 \mathrm{~K}$ to $1000 \mathrm{~K}$.

The ${ }^{111}$ In probe decays by electron capture to an excited state of ${ }^{111} \mathrm{Cd}$ with a mean-life 4.0 dạys. Two $\gamma$-rays are then emitted. There is a well-established anisotropy in the direction of emission of the second radiation with respect to the emission direction of the first one. For two detectors recording $\gamma_{1}$ and $\gamma_{2}$ under a fixed angle $\vartheta$ the coincidence counting rate $N(\vartheta, t)$ is given by [5]

$$
N(\vartheta, t)=N_{0} \mathrm{e}^{-t / \tau}\left(1+A_{2} G_{2}(t) P_{2}(\cos \vartheta)\right),
$$

where $t$ is the time which elapsed between emission of $\gamma_{1}$ and $\gamma_{2}, \tau=120 \mathrm{~ns}$ is the mean-life of the intermediate excited state of ${ }^{111} \mathrm{Cd}$ and $A_{2}=-0.18$ is the angular correlation coefficient. The perturbation factor $G_{2}(t)$ contains all relevant information about the interaction of nuclear moments with extra-nuclear fields. With two start and two stop detectors forming the angle $\vartheta=90^{\circ}$ or $180^{\circ}$ four $N(\vartheta, t)$ spectra were recorded simultaneously. After subtraction of a constant background, the usual ratio 


$$
R(t)=2 \frac{N\left(180^{\circ}, t\right)-N\left(90^{\circ}, t\right)}{N\left(180^{\circ}, t\right)+2 N\left(90^{\circ}, t\right)}=A_{2} G_{2}(t)
$$

was formed using geometrical mean of the $N(\vartheta, t)$ values [6]

The analysis of the $R(t)$ spectra was performed assuming two components corresponding to two different surroundings of ${ }^{111} \mathrm{In}\left({ }^{111} \mathrm{Cd}\right)$ in the alloys. A part $f$ of the probe nuclei was exposed to a unique electric field gradient due to one manganese atom in the nearest neighbour shell of the probe atom. The remaining $(1-f)$ nuclei do not experience any quadrupole interaction as it happens in pure cubic metals. Obviously, these probes have the silver host atoms only in their nearest neighbourhood. In such a case the perturbation factor $G_{2}(t)$ consists of two components and is then given by $[5,7]$

$$
G(t)=f\left[\sum_{n=0}^{3} S_{2 n} \cos \left(\frac{3 \pi}{10} n \nu_{Q} t\right)\right]+(1-f),
$$

where $f$ gives the relative number of the ${ }^{111} \mathrm{In}-\mathrm{Mn}$ pairs in the alloy. The magnitude of the electric field gradient is included in the quadrupole interaction frequency $\nu_{Q}$. The $S_{2 n}$ coefficients are tabulated in Ref. [5]. An example of the measured spectra with the fitted perturbation factor is shown in Fig. 1.

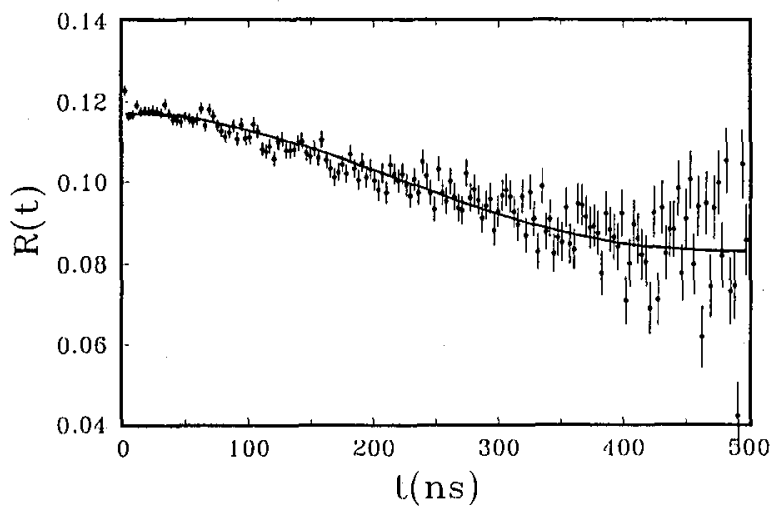

Fig. 1. The PAC spectrum for ${ }^{111} \mathrm{In}$ in $\mathrm{Ag}_{0.99} \mathrm{Mn}_{0.01}$ measured at $900 \mathrm{~K}$.

The value of $\nu_{Q}$ obtained from the least square fitting procedure is equal to $3.6(2) \mathrm{MHz}$ independently of the temperature and the $\mathrm{Mn}$ concentration. In contrary, the fraction $f$ depends on the impurity concentration as well as on the temperature. For spectra taken at $750 \mathrm{~K}$, the fraction $f$ equals $0.39,0.21$ and 0.10 for alloys containing $1,0.5$ and 0.2 at.\% of $\mathrm{Mn}$, respectively. The proportional changes of $f$ confirm the assumption that this fraction describes the relative number of ${ }^{111}$ In-Mn pairs.

With increasing temperature the impurity distribution tends to become random and the number of In-Mn pair changes according to the following formula:

$$
f /(1-f) c=\beta \exp (-E / k T),
$$


where $c$ is the impurity concentration, pre-exponential parameter $\beta$ is related to the change of the entropy due to formation of the impurity pair, $E$ is the binding energy [8].

The binding energy could be extracted even from a single measurement of fraction $f$ performed at given temperature $T$ if the value of parameter $\beta$ is assumed $[3,8]$. However, a more precise way requires performing the measurements at several temperatures for alloys of different impurity concentration. Figure 2 presents the logarithmic plot of the ratio $f /(1-f) c$ versus $1 / T$ for AgMn alloys. The data points lie along one straight line independent of the impurity concentration. The slope of this line gives the binding energy between $\mathrm{Mn}$ and In atoms in silver $E=-44(9) \mathrm{meV}$. Negative sign of $E$ means the attractive interaction between In and $\mathrm{Mn}$ impurities in $\mathrm{Ag}$.

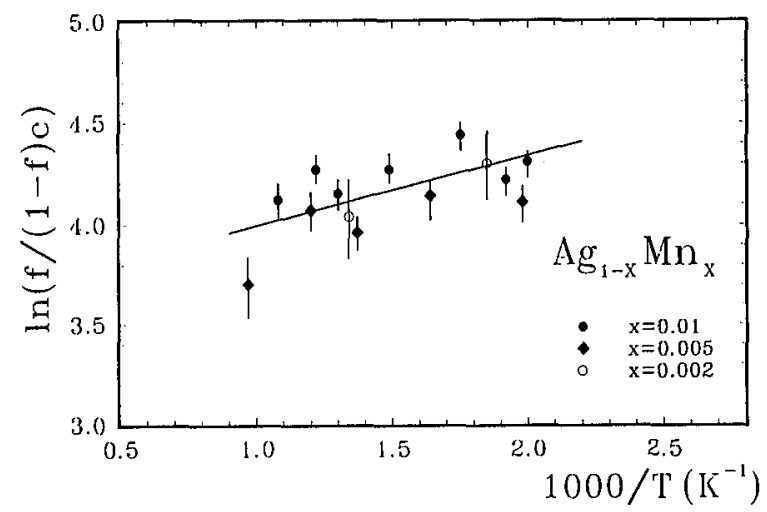

Fig. 2. Logarithmic plot of normalized fraction of the ${ }^{111}$ In probe forming In-Mn pairs in dilute alloys of silver. Solid line is the least squares fit of Eq. (4) with $\beta$ and $E$ as free parameters.

\section{3. ${ }^{57} \mathrm{Co}$ in AgIn dilute alloys}

The ${ }^{57} \mathrm{Co}$ probe atoms were introduced into pure silver and into two AgIn dilute alloys with the indium concentration of 0.3 and 0.6 at\%. The samples were annealed at $1000 \mathrm{~K}, 800 \mathrm{~K}$ and $600 \mathrm{~K}$ for 24 hours and quickly cooled down to the room temperature. After each annealing step one Mössbauer spectrum was taken at room temperature. The investigated samples served as Mössbauer sources while the powder of $\mathrm{K}_{4}\left[\mathrm{Fe}(\mathrm{CN})_{6}\right] \cdot 3 \mathrm{H}_{2} \mathrm{O}$ was used as a single line standard absorber. An example of the spectrum taken with ${ }^{57} \mathrm{Co}$ in $\mathrm{Ag}_{0.994} \mathrm{In}_{0.006}$ is shown in Fig. 3.

The spectrum recorded for ${ }^{57} \mathrm{Co}$ in pure silver has a form of the single line which corresponds to isolated Co atoms in $\mathrm{Ag}$ lattice. In the case of alloys (see Fig. 3) an additional quadrupole doublet appears in the spectra. It is tempting to assign this component to ${ }^{57} \mathrm{Co}$ forming Co-In nearest neighbour pairs with In impurities. The binding energy between Co and In atoms would then be given by Eq. (4) with $f$ being the relative intensity of the quadrupole doublet. Within 


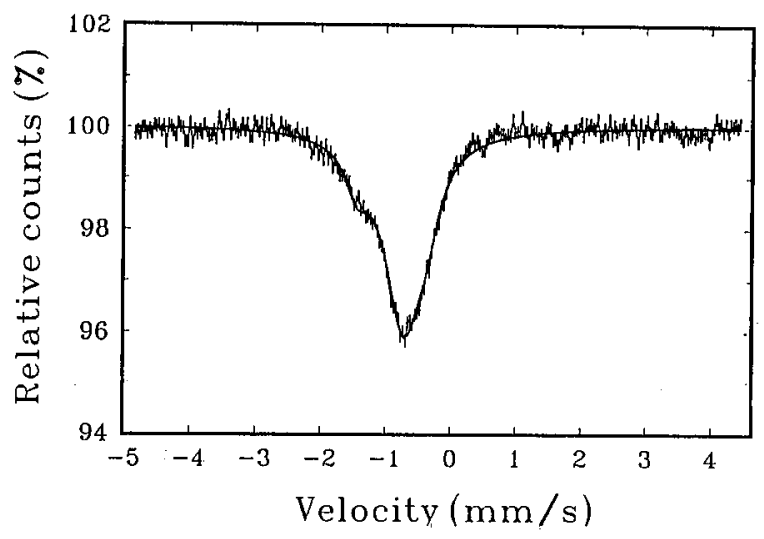

Fig. 3. The Mössbauer spectrum of ${ }^{57} \mathrm{Co}$ in the $\mathrm{Ag}_{0.0994} \operatorname{In}_{0.006}$ alloy measured at room temperature after annealing at $600 \mathrm{~K}$.

this picture the quadrupole splitting is expected to be independent of the alloy concentration and the annealing temperature. This is not the case however. The quadrupole splitting and the isomer shift change with the annealing temperature in the rather nonsystematic way. For example, the fitted value of the quadrupole splitting ranges from -0.41 to $-0.54 \mathrm{~mm} / \mathrm{s}$ for ${ }^{57} \mathrm{Co}$ in $\mathrm{Ag}_{0.994} \mathrm{In}_{0.006}$ alloy and from -0.48 to $-0.66 \mathrm{~mm} / \mathrm{s}$ in $\mathrm{Ag}_{0.997} \mathrm{In}_{0.003}$ alloy. Moreover the quadrupole doublet intensity varies irregularly and the binding energy value calculated separately for each measurement is scattered in the range between -0.06 and $-0.18 \mathrm{eV}$.

The nonsystematic changes in the hyperfine interaction parameters related to the quadrupole doublet suggest that the Co-In pairs are not the only impurity complexes formed in the system. First of all, formation of Co-In complexes containing more than one In atom leads to the new spectral components with the quadrupole splitting and the isomer shift different from that for the Co-In pair. Another problem arises since cobalt is not soluble in silver. Despite of their extremely low concentration cobalt atoms may aggregate. In fact, small complexes of cobalt atoms may generate broadening observed in the spectra for pure silver. In principle, in the case of ${ }^{57} \mathrm{Co}$ belonging to clusters of different size and structure, an electric field gradient distribution is expected. Unfortunately, the resonance line width is not narrow enough to allow a distinction between different spectral components and consequently between different impurity complexes. Therefore, we are not able to give a precise interpretation of the quadrupole interaction doublets and to conclude unambiguously the binding energy value. The qualitative result is an existence of the attractive interaction between Co and In impurities in silver.

\section{Discussion}

Figure 4 shows how the binding energy for In-X pairs in silver changes its sign from positive for $\mathrm{X}=\mathrm{Ga}$ and $\mathrm{Zn}$ to negative for $\mathrm{X}=\mathrm{Cu}$ and $\mathrm{Ni}$. We have demonstrated above that the sign remains negative for $\mathrm{X}=$ Co and $\mathrm{Mn}$. This 
tendency is very well reproduced by the model in which the binding energy is discussed in terms of the creation and the break-up of the impurity-impurity and impurity-host bonds [9]. The energy of a single bond was referred to the heat of

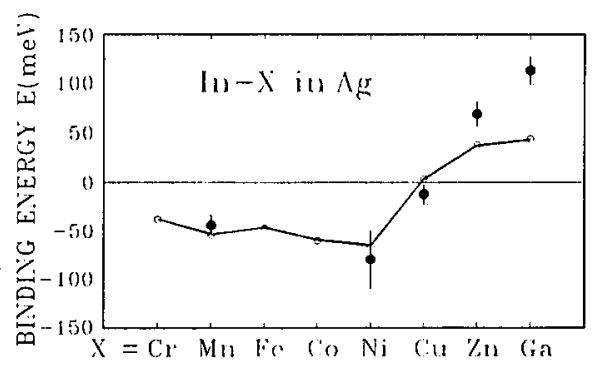

Fig. 4. Binding energies of In-impurity pairs in Ag. The measured In-Mn value is presented together with the data published in Refs. [3] and [4]. The solid line represents $E$ calculated using the Miedema parameters according to the procedure described in Ref. [11].
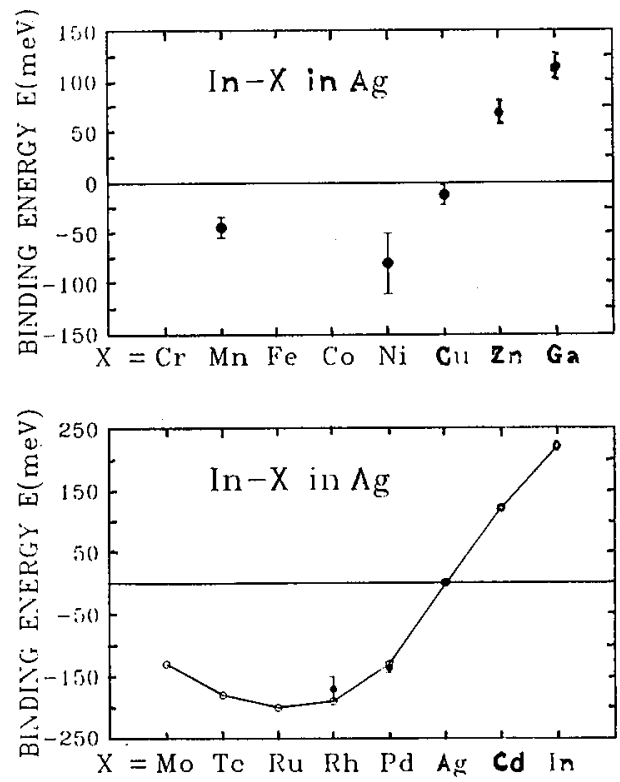

Fig. 5. Impurity-impurity interaction energies. a) The experimental values of the interaction energies of $3 d$ and $4 s p$ impurities with In in $\mathrm{Ag}$ (upper part). b) The results of the KKR calculations [2] of the interaction energies (o) of $4 d$ and $5 s p$ impurities with In in $\mathrm{Ag}$ compared with experimental values ( $\bullet$ ) [1] (lower part).

formation for corresponding alloys and then calculated on the basis of the semiempirical model developed by Miedema et al. [10]. The solid line in Fig. 4 represents 
the values calculated in the way formulated by Cranshaw [11]. The experimental and calculated values agree surprisingly well in particular for $d$-metal impurities. A small discrepancy for In- $\mathrm{Zn}$ and In-Ga pairs is understood, since according to the authors [12] the Miedema parameters are not practical in predicting the heat of formation for alloys of two nontransition metals.

A substantial progress has been made recently in the first principle calculations of the nearest neighbour interaction energies for impurity pairs in $\mathrm{Ag}$ and $\mathrm{Pd}$ crystals [2]. The calculations were based on the local-density theory and apply the KKR Green's function method for spherical potentials. The calculated energies are in very good agreement with the binding energies determined by PAC experiments for In-Rh and In-Pd impurity pairs in Ag. This is shown in Fig. 5b together with the results of the calculations for In-X pairs with $\mathrm{X}$ being the $4 d$ and $5 s p$ impurity atoms from Mo to In. With two above-mentioned exceptions the binding energy could not be measured for these impurity pairs in $\mathrm{Ag}$ because of a solid solubility limitation for $4 d$ elements and an absence of the nearest neighbour PAC signal for ${ }^{111} \mathrm{In}$ in silver based alloys with $5 s p$ impurities [1]. As it is shown in Fig. 5a much more complete experimental results are now available for $3 d$ and $4 s p$ impurities. Both, the experimental binding energy plotted in Fig. 5a and the calculated values plotted in Fig. $5 \mathrm{~b}$ vary in a similar way. This result is very encouraging. We hope the present data will serve as a quantitative test for current theoretical calculations.

\section{Acknowledgment}

This work was supported by Research Grant KBN PB 2594/2/91 to Jagiellonian University.

\section{References}

[1] K. Królas, Hyperfine Interactions 60, 581 (1990).

[2] T. Hoshino, B. Drittler, R. Zeller, P.H. Dederichs, Phys. Rev. B 45, 12202 (1992).

[3] M. Sternik, K. Królas, Phys. Rev. B 40, 4171 (1989).

[4] M. Sternik, K. Królas, Phys. Status Solidi B 169, 323 (1992).

[5] H. Frauenfelder, R.M. Steffen, in: Alpha-, Beta-, Gamma-Ray Spectroscopy, Vol. 2, Ed. K. Sieghbahn, North-Holland, Amsterdam 1974, p. 997.

[6] A.R. Arends, C. Hohenemser, F. Pleiter, H. deWaard, I. Chow, R.M. Suter, Hyperfine Interactions 8, 191 (1980).

[7] G. Schatz, A. Weindinger, Nukleare Festkörperphysik, Teubner, Stuttgart 1985.

[8] A.Z. Hrynkiewicz, K. Królas, Phys. Rev. B 28, 1864 (1983).

[9] K. Królas, Phys. Lett. A 85, 107 (1981).

[10] A. Miedema, A.K. Niessen, CALPHAD 7, 27 (1983).

[11] T.E. Cranshaw, J. Phys. F, Met. Phys. 17, 1645 (1987).

[12] F.R. Boer, R. Boom, W.C.M. Mattens, A. Miedema, A.K. Niessen, in: Cohesion in Metals, Eds. F.R. Boer, D.G. Pettifer, North-Holland, Amsterdam 1988, p. 56. 